\title{
Analysis of Edge Detection Technique by Varying Image Contrast
}

\author{
Tasnuva Tasneem ${ }^{1}$, Zeenat Afroze ${ }^{2}$ \\ ${ }^{1}$ (Lecturer, EEE Department, American International University-Bangladesh) \\ ${ }^{2}$ (Assistant Professor, EEE Department, American International University-Bangladesh)
}

\begin{abstract}
Edge detection technique is a vital part of image processing that can be applied to different types of images including medical images. Contrast adjustment is another important part of image processing which can have significant effect on the edge detection process. This paper discusses about edge detection technique along with the algorithm. Detailed analysis based on edge detection for images of different contrast has been performed. Results are analyzed corresponding to images of different contrast which can be achieved by histogram stretching.
\end{abstract}

Keyword: Contrast adjustment, Edge detection, Image processing, Medical image, Visual quality

\section{Introduction}

Edge detection has become a very important part of digital image processing now-a-days. It is one of the most critical tasks in automatic image analysis [1]. Image edge detection significantly reduces the amount of data and filters out useless information, while preserving the important structural properties in an image [2]. Edge detection, a fundamental tool in image processing, has an immense application in feature detection and feature extraction. Edge detection technique can be also effectively applied to different medical images. Medical images edge detection is an important work for object recognition of the human organs and it is an important pre-processing step in medical image segmentation and 3-D reconstruction [3]. Contrast adjustment is another vital part of image processing. Contrast of an image can be determined by the ratio between the brightest and the darkest pixel intensities [4]. Contrast adjustment helps to extract maximum information from an image. Extracting maximum information from a source image results in a processed image which is more suitable for human vision. In this paper, edge detection method is applied on images of different contrast. An effective technique called histogram stretching is used here for adjusting the contrast of images. The rest of the paper is arranged as follows-

Detailed explanation based on contrast adjustment and edge detection is given in section 2. Section 3 explains the methodology that has been used to perform the analysis. The results and analysis are shown in section 4. Section 4.1 includes edge detection of real city scan images. Comparisons are shown by varying the pixel removal while adjusting the contrast for the images. Section 4.2 shows edge detection for images of some other aspects. Section 5 and 6 include the discussion and conclusion respectively.

\subsection{Contrast Adjustment}

\section{Literature Review}

Many real world images are acquired with low contrast and unsuitable for human eyes to read [5]. So the contrast of those images needs to be improved. For the adjustment of image contrast, the background and the objects of an image should be distinguished properly. The light colors are needed to be made lighter and the dark colors are needed to be made darker. It can be done by setting all color components below a specified lower bound to zero, and all color components above a specified upper bound to the maximum intensity (that is, 255) [6]. A method called histogram stretching is used here for adjusting the image contrast. A histogram represents the frequency of occurrence of all the gray levels in an image [7]. Two operations are performed for stretching the histogram. Firstly, a value is added to all the pixels which basically add that amount of value to the histogram. It shifts the histogram visually. Secondly, all the pixel values are multiplied by a certain amount of scales where the histogram data appears, which stretches the histogram visually. Figure shows how the histogram is stretched for adjusting the contrast (Fig 1). Here, the left-most non-zero bin is mapped to zero and the right-most non-zero bin is mapped to 255 in the histogram for obtaining the stretching. This stretching improves the image contrast by making the light toned areas appearing lighter and dark areas appearing darker. This kind of contrast adjustment makes visual interpretation much easier. 


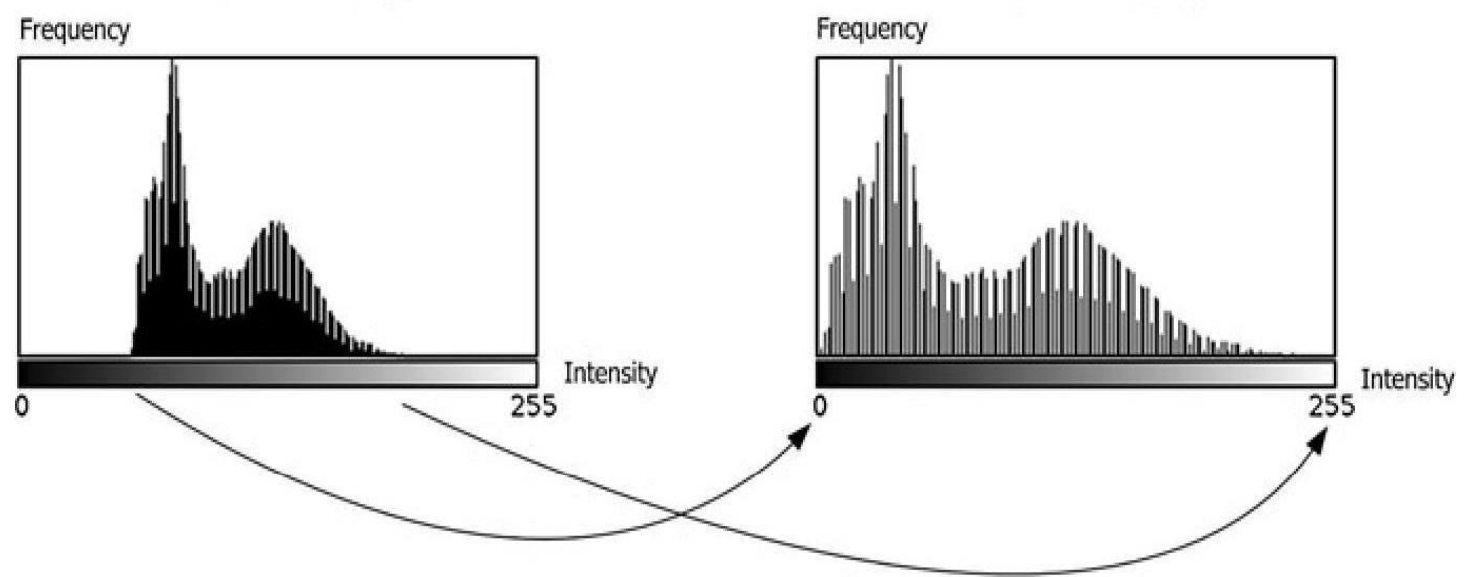

Fig 1: Stretching of histogram for adjusting image contrast

\subsection{Edge Detection}

An edge can be defined as an area where image intensity changes significantly. More precisely edge is the boundary between the background and objects of an image. By locating the areas with strong intensity contrast, edge can be detected. For detecting edges of an image, firstly, color images have to be converted to gray scale images. After that detection method has to be applied. For converting a color image to a gray scale image, any of the three methods can be used, which are - lightness method, average method and luminosity method. Each color pixel is described by a triple $(R, G, B)$ of intensities for red, green and blue.

The lightness method averages the most prominent and least prominent colors: $(\max (R, G, B)+\min (R, G, B)) / 2$ The average method simply averages the values: $(\mathrm{R}+\mathrm{G}+\mathrm{B}) / 3$

The luminosity method is a more sophisticated version of the average method. It also averages the values, but it forms a weighted average to account for human perception. Human vision is more sensitive to green than the other colors, so green is weighted most heavily. The formula for luminosity is: $0.21 \mathrm{R}+0.72 \mathrm{G}+$ $0.07 \mathrm{~B}$

After this conversion, edge detection method can be applied. The most frequent detection methods are explained in the following.

The Roberts Detection: The Roberts Cross operator performs a simple, quick to compute, 2-D spatial gradient measurement on an image, thus highlights regions of high spatial frequency which often correspond to edges[8].

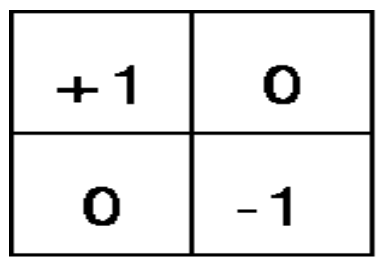

$G \times$

Fig 2: Robert's Mask

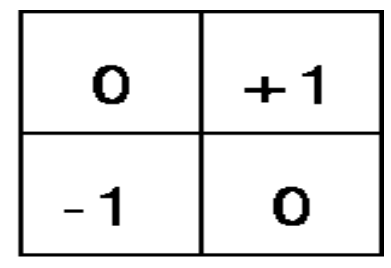

Gy

The Prewitt Detection: The Prewitt edge detection is an appropriate way to estimate the magnitude and orientation of an edge[8].

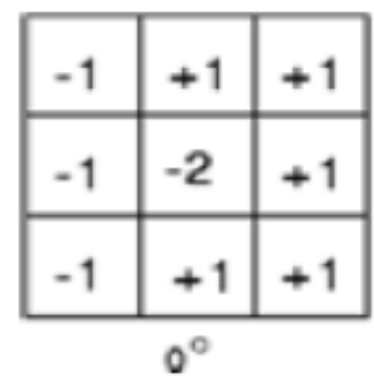

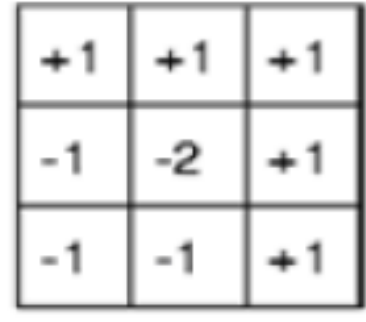

$45^{\circ}$

Fig 3: Prewitt Mask

The Sobel Detection: The Sobel operator performs a 2-D spatial gradient measurement on an image and so emphasizes regions of high spatial frequency that correspond to edges [8]. 


\begin{tabular}{|c|c|c|}
\hline-1 & 0 & +1 \\
\hline-2 & 0 & +2 \\
\hline-1 & 0 & +1 \\
\hline
\end{tabular}

$G \times$

\begin{tabular}{|c|c|c|}
\hline+1 & +2 & +1 \\
\hline 0 & 0 & 0 \\
\hline-1 & -2 & -1 \\
\hline
\end{tabular}

Gy

Fig 4: Sobel Mask

\section{Methodology}

Edge detection technique, an important part of image processing, has been analyzed here. Edge detection has been performed using the Robert detection, Prewitt detection and Sobel detection which are explained in details in section 2.2. Before the detection conversion of color images to gray scale images has been performed using the luminosity method which is also mentioned in section 2.2. The detection method has been analyzed by varying the image contrast. An efficient method named as histogram stretching is used here for varying the contrast as stated in section 2.1. Contrast adjustment differs depending upon the amount of histogram shifting, which means the amount of pixel removal. Images of different contrast are obtained here by changing the amount of pixel removal starting from $0 \%$ to $100 \%$. Results are obtained and analyzed by applying edge detection method to images of different contrast.

\section{Results and Analysis}

Image contrast has been adjusted by histogram stretching firstly, and then edge detection technique has been applied to the images of different contrast.

\subsection{Edge detection applied to medical Images}

Image processing techniques have been developed for analyzing the output of medical imaging system to get the advantage to analyze the symptoms of the patients with ease [9]. The images used here are real city scan images collected from 'Shaheed Suhrawardy Hospital' in Bangladesh. Two city scan images are used here taken from the abdominal area of a person. One is representing the transverse cut section whereas other the longitudinal cut section. Edge has been detected after adjusting the image contrast for different amount of pixel removal. For both of the city scan images, the amount of pixel removal has been varied from $0 \%$ to $100 \%$. Results show that for $0 \%$ to around $38 \%$, the improvement of image contrast enhances the visual quality of the images. After $40 \%$, the increase of contrast rather degrades the visual quality because of excessive pixel removal.

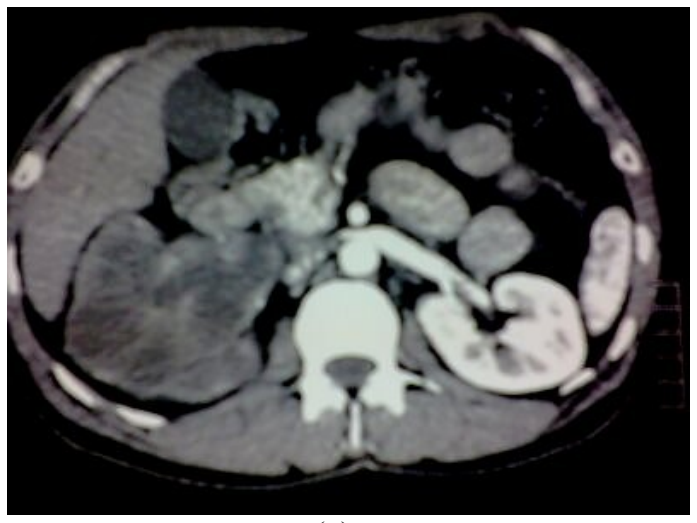

(a)

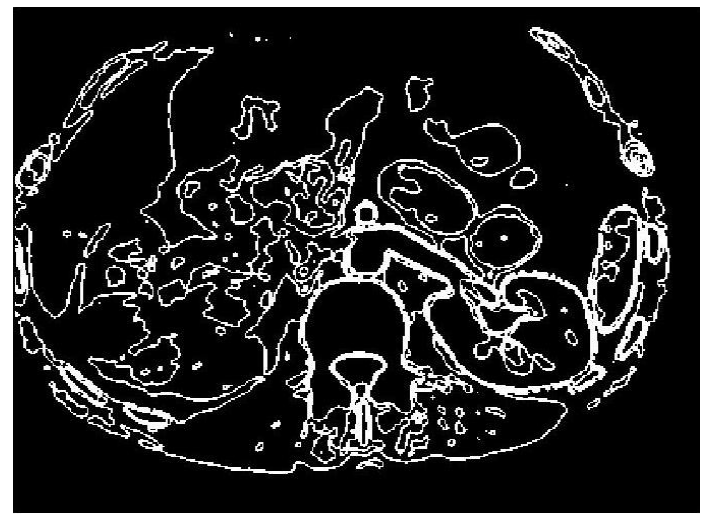

(b)

Fig 5: Edge detection of an original city scan image taken from abdominal area (transverse cut section)

(a) Source image (b) Detected edges 


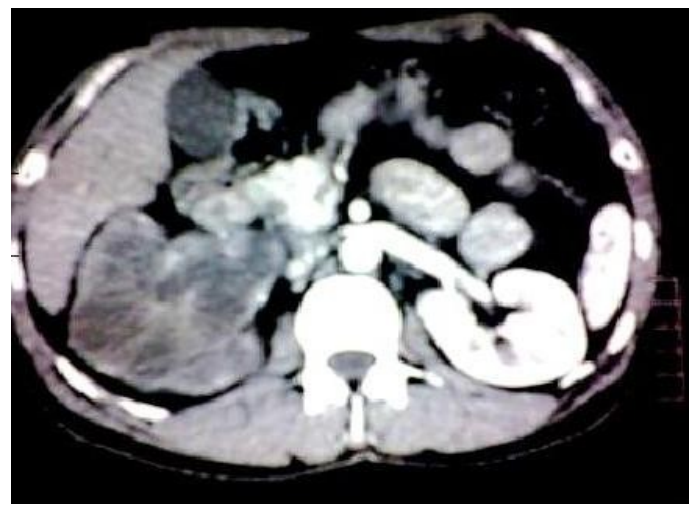

(a)

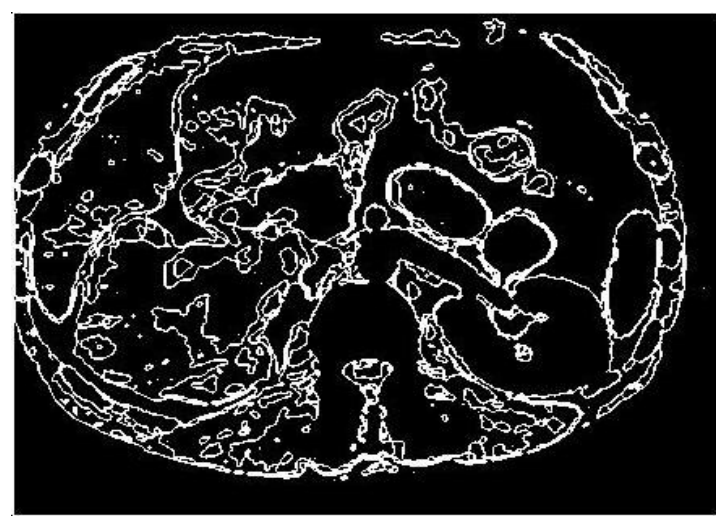

(b)

Fig 6: Edge detection after increasing contrast by $10 \%$ pixel removal from the original city scan image taken from abdominal area (transverse cut section) (a) Source image (b) Detected edges

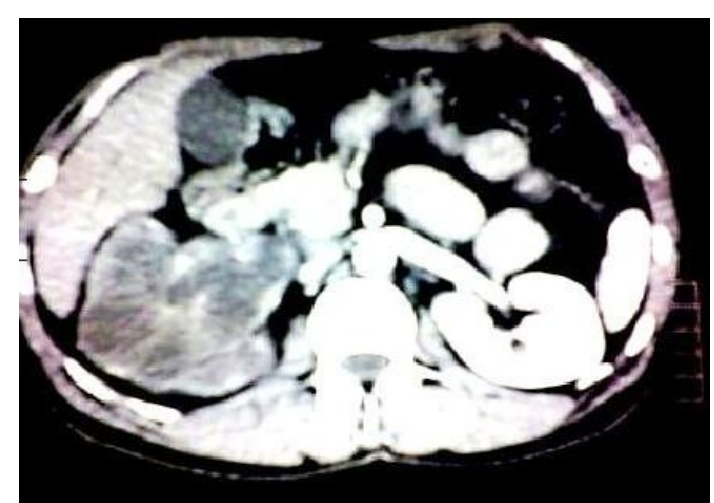

(a)

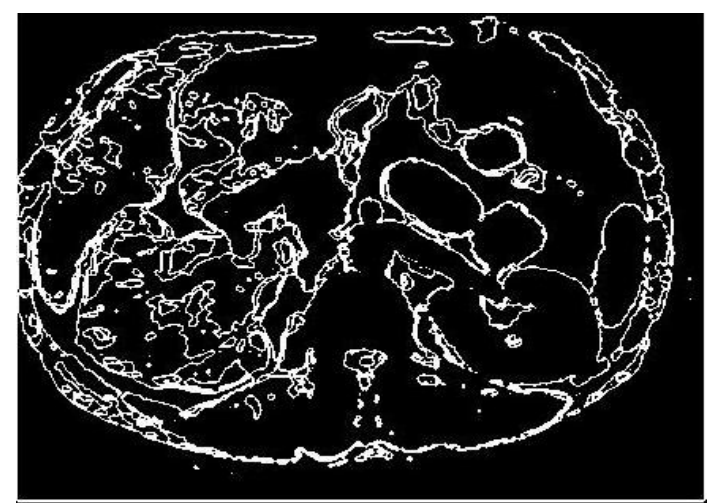

(b)

Fig 7: Edge detection after increasing contrast by $20 \%$ pixel removal from the original city scan image taken from abdominal area (transverse cut section) (a) Source image (b) Detected edges

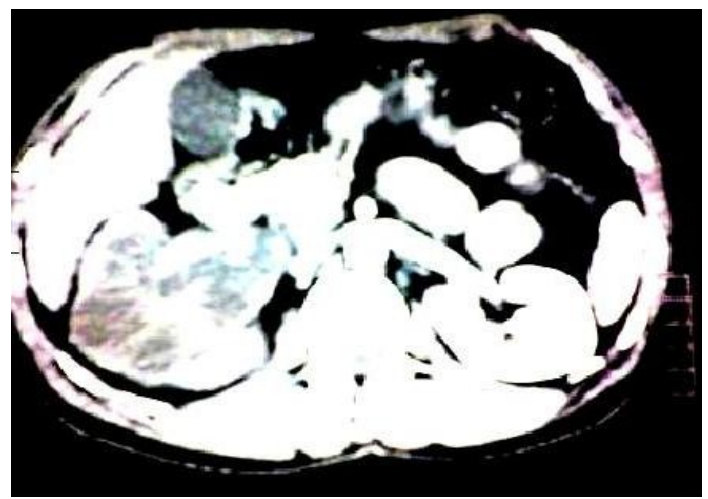

(a)

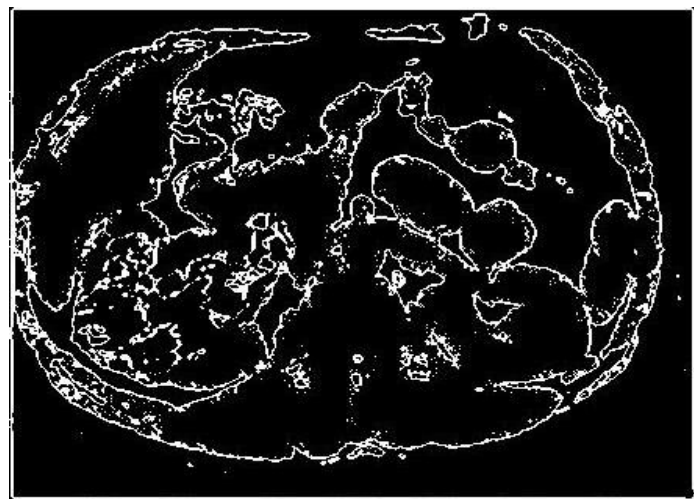

(b)

Fig 8: Edge detection after increasing contrast by $40 \%$ pixel removal from the original city scan image taken from abdominal area (transverse cut section) (a) Source image (b) Detected edges 


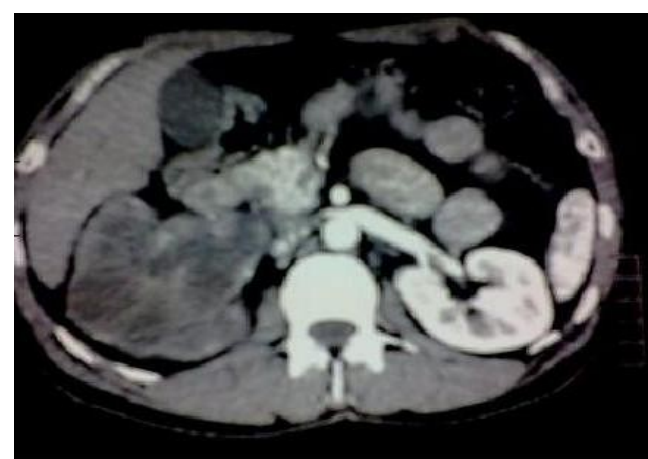

(a)

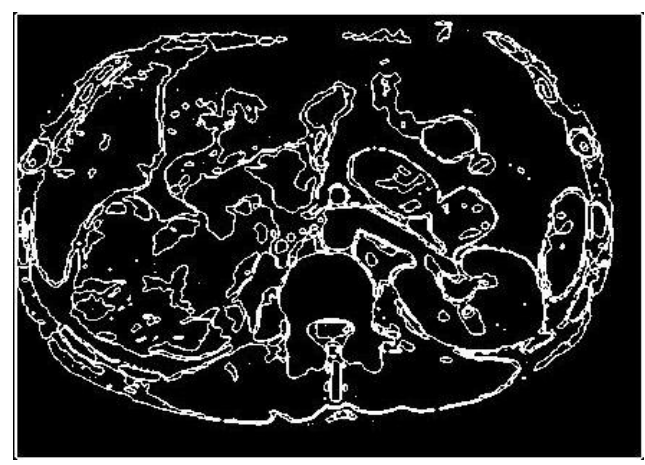

(b)

Fig 9: Edge detection after increasing contrast by $60 \%$ pixel removal from the original city scan image taken from abdominal area (transverse cut section) (a) Source image (b) Detected edges

The visual quality of the edge detected images is enhanced as the amount of pixel removal as well as the image contrast is improved. But when it reaches around $40 \%$ pixel removal, the image becomes slightly distorted. This happens because of too much stretching of the intensity histogram which causes excessive information removal. As a result the accuracy of edge detection also reduces because the source image is distorted. But after 50\%, it again tends to increase the accuracy of the detection because the contrast adjustment is better.

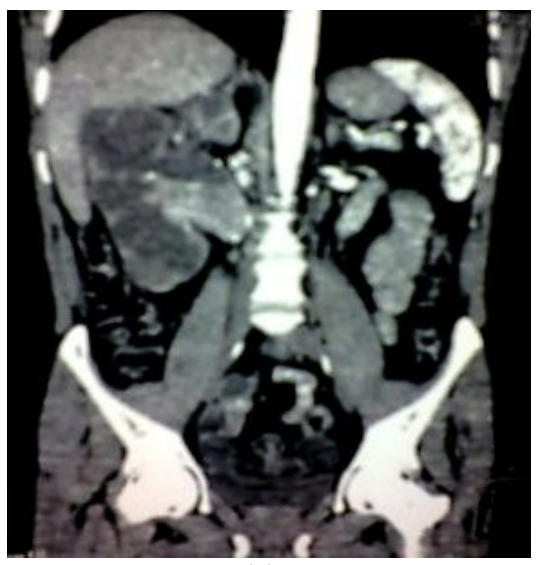

(a)

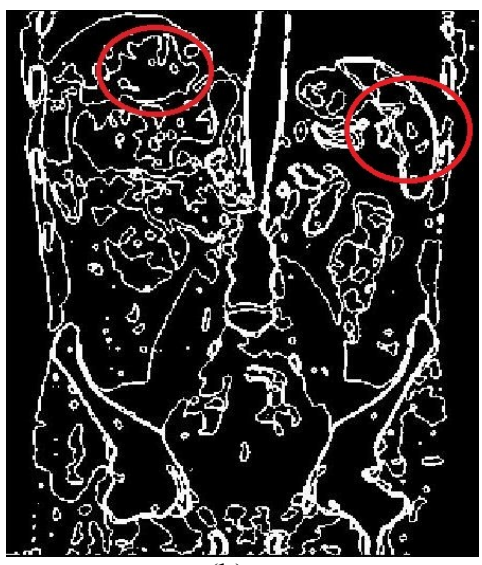

(b)

Fig 10: Edge detection of an original city scan image taken from abdominal area (longitudinal cut section)

(a) Source image (b) Detected edges

The red marked portion (Fig 10-b) is representing a detection of edges which is not desired as compared with the source image (Fig 10-a). This can be improved by adjusting the contrast of the used medical image. The improved source image as well as the corrected edge detected image are given in the following (Fig 11).

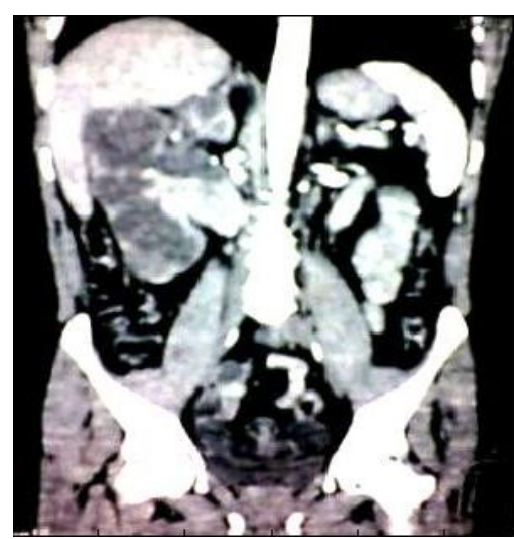

(a)

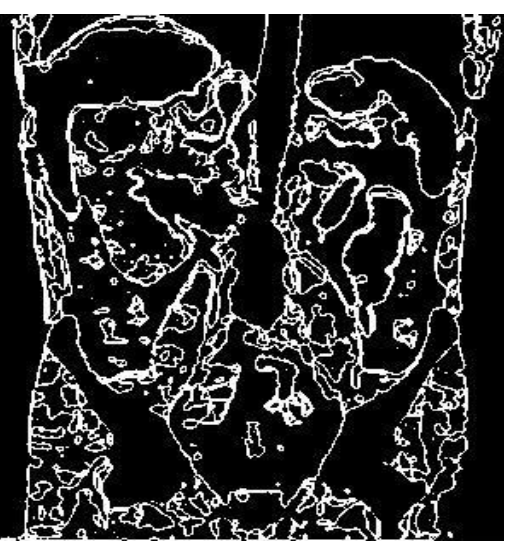

(b)

Fig 11: Edge detection after increasing contrast by $20 \%$ pixel removal from the original city scan image taken from abdominal area (longitudinal cut section) (a) Source image (b) Detected edges 
4.2 Edge detection applied to images of different aspects

In this section, edge detection has been performed for images of different aspects varying the contrast.

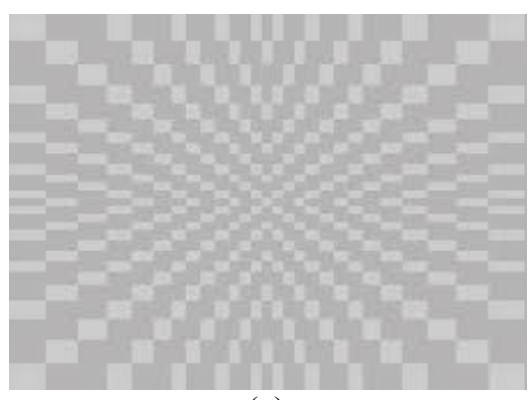

(a)

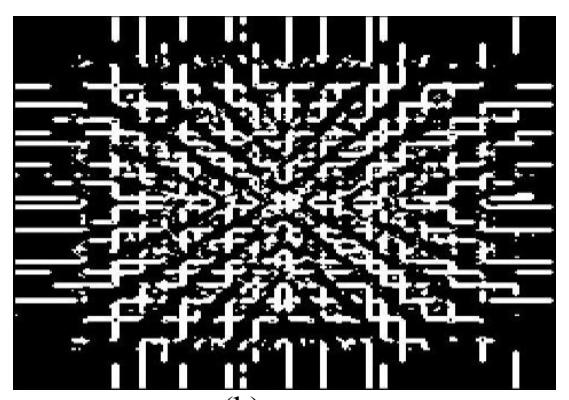

(b)

Fig 12: Edge detection of an image representing a random pattern (a) Source image (b) Detected edges

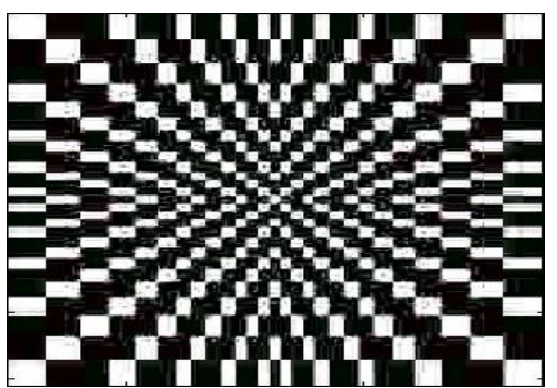

(a)

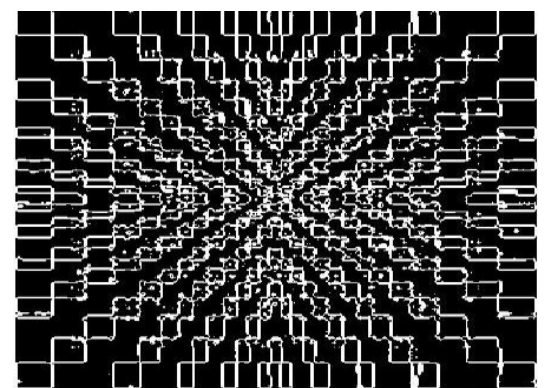

(b)

Fig 13: Edge detection after increasing contrast by $20 \%$ pixel removal from image representing random pattern (a) Source image (b) Detected edges

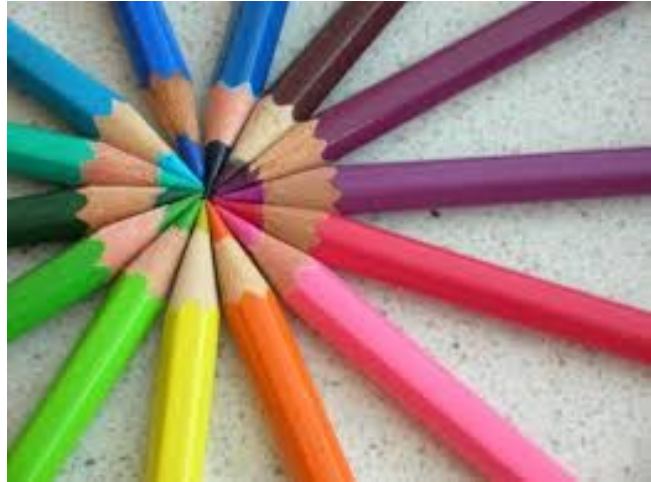

(a)

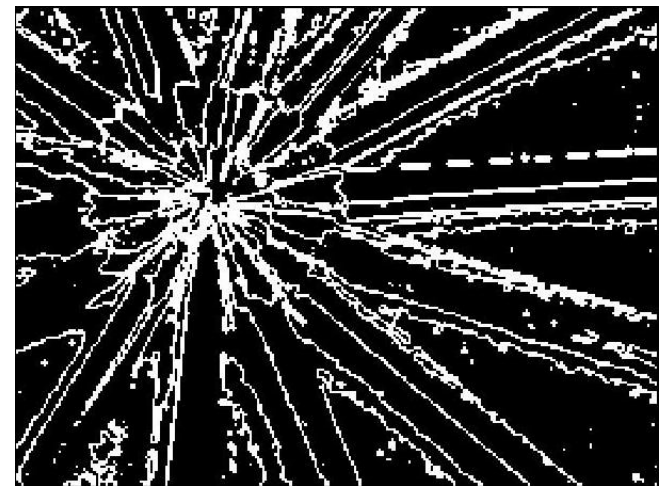

(b)

Fig 14: Edge detection of a color image (a) Source image (b) Detected edges

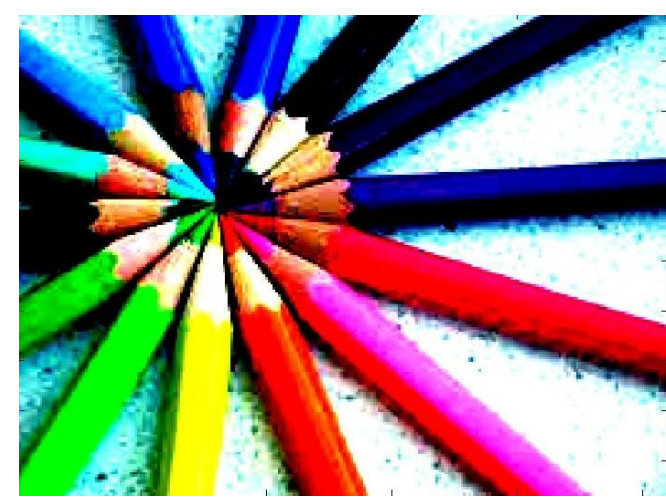

(a)

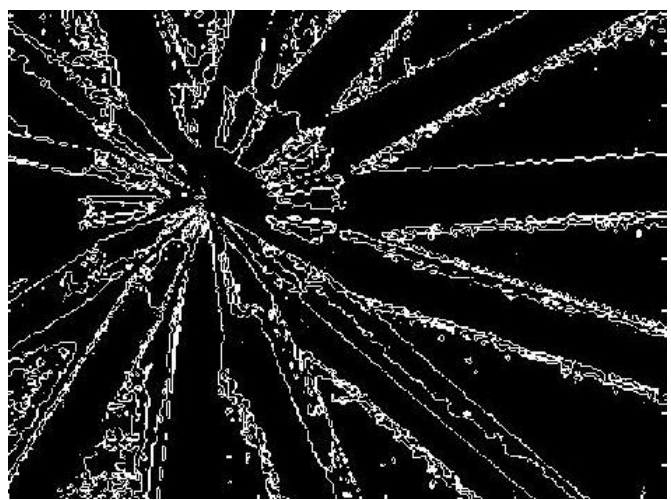

(b)

Fig 15: Edge detection after increasing contrast by $35 \%$ pixel removal from a color image (a) Source image (b) Detected edges 
It can be noticed that if edge is detected after increasing the image contrast, it gives more accuracy in the detection. Here the percentage of pixel removal is taken as $20 \%$ (Fig 13) and 35\% (Fig 15).

\section{Discussion}

Edge detection requires identifying the boundaries between object and the background of an image, whereas contrast adjustment ensures the object and the background to be distinguished. This relation leads to a decision that, the more accurate will be the contrast of an image, the more accurate will be the result of the edge detection technique applied to different images.

\section{Conclusion}

This paper includes improving the quality of an image by adjusting image contrast and applying edge detection technique on images of various contrasts. Analysis and comparison have been shown for edge detection method on images of different contrast. In this paper, only the image contrast has been varied. Parameters other than the image contrast can be varied and analyzed further. The accuracy in the image contrast is used for the accuracy in edge detection. This improvement can be used in medical image pre-processing for diagnosis by detecting affected cells from normal cells. It can also be applied to any other images for improving the visual quality.

\section{References}

[1] Mohamed A. El-Sayed, A New Algorithm Based Entropic Threshold for Edge Detection in Images, International Journal of Computer Science Issues, Vol. 8, Issue 5, No 1, September 2011

[2] Raman Maini and Dr. Himanshu Aggarwal, Study and Comparison of Various Image Edge Detection Techniques, International Journal of Image Processing, Vol. 3, Issue 1

[3] Anurag Sharma, Pankaj Sharma, Rashmi and Hardeep Kumar, Edge Detection of Medical Images Using Morphological Algorithms, International Journal for Science and Emerging Technologies with Latest Trends, Vol. 4, Issue 1

[4] Dr. Muna F. Al-Samaraie, A New Enhancement Approach for Enhancing Image of Digital Cameras by Changing the Contrast, International Journal of Advanced Science and Technology, Vol. 32, July 2011

[5] Sukhjinder Singh, R.K. Bansal and Savina Bansal, Comparative Study and Implementation of Image Processing Techniques Using Matlab, International Journal of Advanced Research in Computer Science and Software Engineering, Vol. 2, Issue 3, March 2012

[6] Manvi, Rajdeep Singh Chauhan and Manpreet Singh, Image Contrast Enhancement Using Histogram Equalization, International Journal of Computing and Business Research, 2012

[7] Er. Vinod Saini and Er. Tarun Gulati, A Comparative Study on Image Enhancement Using Image Fusion, International Journal of Advanced Research in Computer Science and Software Engineering, Vol. 2, Issue 10, October 2012 (141-145)

[8] Matheel E. Abdulmunim and Suhad M., Propose a Mixture Edge Detection Method for Infrared Image Segmentation, British Journal of Science, Vol. 6, Issue 2, September 2012 (61-72)

[9] Gautam Appasaheb Kudale and Mahesh D. Pawar, Study and Analysis of Various Edge Detection Methods for X-Ray Images, International Journal of Computer Science and Application, 2010 (15-17) 